\title{
Didacticiel d'instrumentation virtuelle à coût réduit
}

\author{
Jean MBIHI \\ E-mail : mbihidr@yahoo.fr \\ N. KELAKO KENADJA \\ E-mail: nathankanedja@yahoo.fr \\ M. L. NGO NOUCK \\ E-mail : masendma@yahoo.fr \\ Groupe de Recherche en Informatique Industrielle et Automation (GR2IA), \\ École Normale Supérieure d'Enseignement Technique (ENSET) \\ BP 1872, Douala, Cameroun.
}

\section{Résumé}

Cet article présente un didacticiel d'instrumentation virtuelle, développé pour le cycle de Master en génie électrique à l'ENSET de Douala. La partie matérielle dudit didacticiel est constituée en amont d'un générateur de signaux basses fréquences, suivi d'un multiplexeur de sélection du signal à acquérir et d'un convertisseur $\mathrm{A} / \mathrm{N}$ relié en aval au connecteur DB 25 $\mathrm{du}$ port LPT d'un ordinateur PC. Le logiciel d'instrumentation virtuelle prévu pour l'exploitation du didacticiel a été développé en Visual Basic et bien testé sous Windows 9.X/Me/2000/XP. L'intérêt du didacticiel proposé en informatique industrielle, est fondé sur la simplicité structurelle, le coût réduit, la facilité d'utilisation, et la possibilité de sauvegarder tous les résultats obtenus sur disque sous forme d'un fichier à extension.$M$, ce qui permet d'analyser directement les résultats d'instrumentation obtenus avec Matlab.

Mots clés: Didacticiel, générateur de signaux, instrumentation virtuelle, informatique industrielle, Matlab.

Niveau des étudiants : Le didacticiel présenté est utilisé par les étudiants de niveau Master de l'ENSET de Douala, dans le cadre des travaux pratiques d'instrumentation et de traitement du signal par ordinateur.

Niveau de connaissances requis : Électronique, traitement du signal (filtres numériques), programmation système, introduction à Matlab.

\section{Introduction}

Le didacticiel présenté ici a été conçu et réalisé au sein du Groupe de Recherche en Informatique Industrielle et Automation (GR2IA) de l'ENSET de Douala. Il est utilisé dans les filières génie électrique et génie informatique, comme plate-forme didactique d'appoint de travaux pratiques d'instrumentation virtuelle par ordinateur. À la fin de ces travaux pratiques, les étudiants ayant bien exploités le didacticiel, seront capables de :

- Maîtriser l'architecture de l'interface matérielle de génération de signaux multiples et de conversion $\mathrm{A} / \mathrm{N}$ multiplexée.

- Comprendre les algorithmes utilisés pour le développement des procédures événementielles d'acquisition et de filtrage numérique des signaux. 
- Connaître les problèmes techniques rencontrés lors de la mise en œuvre de ces algorithmes (accès aux ports matériels sous Windows NT/2000/XP, génération de période d'échantillonnage, etc.) ainsi que les solutions correspondantes utilisées.

- Vérifier et consolider le théorème d'échantillonnage par l'observation d'un signal acquis pour un choix particulier d'une période d'échantillonnage ne respectant pas ce théorème.

- Migrer tous les résultats obtenus vers Matlab en vue d'accéder aux fonctions d'analyse numérique avancées.

Les prochaines sections de l'article qui permettront de comprendre les bases de construction ainsi que l'utilisation du didacticiel d'exploitation proposé, sont organisées comme suit :

- Interface matérielle.

- Logiciel d'instrumentation virtuelle.

- Travaux pratiques à l'aide du didacticiel.

- Conclusion.

- Références bibliographiques.

\section{Interface matérielle}

\subsection{Schéma synoptique}

Une structure acceptable de l'interface matérielle à proposer devrait satisfaire à de multiples contraintes parmi lesquelles :

- La simplicité de mise en œuvre.

- La facilité de maintenance.

- Le coût réduit.

- La facilité de programmation.

Face à ces contraintes à satisfaire, nous avons orienté notre réflexion vers les solutions d'interfaçage par port LPT. Le schéma synoptique retenu pour l'interface matérielle est représenté à la figure 1.

\section{Figure 1. Schéma synoptique de l'interface d'entrée}

Ce schéma est constitué de deux principales parties qui sont:

- Une carte d'interface matérielle pour port LPT qui intègre les modules électroniques ci-après :

○ Générateur de signaux basses fréquences (sinus, carré, triangle) à fréquence variable.

- Un Multiplexeur analogique $(\mathrm{MuX})$ adressable par programmation, permettant de choisir le type de signal désiré.

○ Un amplificateur du signal sélectionné, à gain réglable.

- Un convertisseur $\mathrm{A} / \mathrm{N}$ de 8 bits, qui convertit le signal sélectionné.

$\circ$ Un multiplexeur de bus de type 8/4 à adressage programmable, permettant de décomposer et de transférer au bus d'état du port LPT, le mot de 8 bits disponible à la sortie du convertisseur $\mathrm{A} / \mathrm{N}$, en deux quartets successifs qui sont ensuite combiner de façon logicielle pour reconstituer instantanément l'octet initial.

- Un connecteur et un câble parallèle DB-25 permettant de relier l'interface matérielle au port LPT de l'ordinateur. 
- Un ordinateur PC équipé d'un port LPT et contenant le logiciel d'instrumentation virtuelle.

Le didacticiel ainsi proposé permet de générer des signaux basses fréquences (sinus, triangle, carré), puis d'acquérir et de traiter ces signaux par ordinateur.

\subsection{Schéma de principe}

La figure 2 correspond au schéma de principe de l'interface d'entrée. Les caractéristiques techniques des composants utilisés pour la mise en œuvre des modules électroniques retenues sont disponibles dans la référence [Int-1].

\section{Figure 2. Schéma de principe de l'interface d'entrée}

Les modules électroniques constitutifs de l'interface matérielle sont :

- Génération de signaux réalisé avec le circuit intégré ICL8038. La fréquence des signaux générés est réglable par un potentiomètre dûment gradué en $\mathrm{Hz}$;

- Multiplexage analogique à l'aide du circuit intégré 74HC4051;

- Amplification du signal grâce à l'amplificateur opérationnel (1/2)-TL072 à gain réglable par potentiomètre dûment gradué en Volts;

- Conversion A/N à l'aide du circuit intégré ADC0820;

- Multiplexage de bus à l'aide du circuit intégré 74LS157;

- Liaison à l'ordinateur par câble normalisé pour port LPT d'un PC. Le brochage et la structure logicielle des registres d'un port LPT d'un PC sont décrits dans les références [1] et [Int-2].

Du point de vue logicielle, chaque octet de données disponible en lecture à la sortie du circuit ADC0820 est donc reconstitué instantanément à partir des lectures instantanées successives de ses deux quartets constitutifs.

\subsection{Maquette du didacticiel}

Le typon de circuit imprimé de l'interface matérielle a été conçu à l'aide du logiciel de CAO électronique Proteus. Ensuite, le circuit imprimé a été réalisé et l'ensemble des organes constitutifs (y compris le module d'alimentation stabilisée et régulée) a été monté sous forme d'une maquette didactique.

Les prises de vue sont illustrées à la figure 3. Les informations détaillées au sujet de la réalisation de la maquette sont disponibles dans [2].

\section{Figure 3. Prises de vue de la maquette didactique}

\section{Logiciel d'instrumentation virtuelle}

\subsection{Fonctions du logiciel}

Le logiciel d'instrumentation virtuelle développé pour contrôler l'interface matérielle du didacticiel offre les fonctionnalités suivantes :

- Choix du type de signal désiré : sinus, triangle, ou carré.

- Acquisition de $\mathrm{N}$ échantillons du signal choisi. 
- Choix et traitement d'un signal acquis $\{\mathrm{S}(\mathrm{n})\}$, à l'aide d'un filtre numérique à réponse impulsionnelle infinie décrit par une équation de récurrence de la forme :

$$
\begin{aligned}
- & \mathbf{s}(0)=\mathbf{y}(0), \quad \mathbf{s}(1)=\mathrm{b}_{0} \mathbf{y}(0)+\mathrm{b}_{1} \mathbf{s}(1)-\mathrm{a}_{1} \mathbf{s}(0), \\
\text { - } & \mathbf{s}(2)=\mathrm{b}_{0} \mathbf{y}(0)+\mathrm{b}_{1} \mathbf{y}(1)+\mathrm{b}_{2} \mathbf{y}(2)-\mathrm{a}_{1} \mathbf{s}(1)-\mathrm{a}_{2} \mathbf{s}(0) ; \\
\text { - } & \mathbf{s}(\mathrm{n})=b_{0} y(n)+b_{1} y(n-1)+b_{2} y(n-2)+b_{3} y(n-3)-a_{1} s(n-1)-a_{2} s(n-2)-a_{3} s(n-3) \\
& \quad \text { pour } n=3,4, \ldots, \mathrm{N}-1 .
\end{aligned}
$$

- Saisie des coefficients $a_{i}(i=1,2,3)$ et $b_{j}(j=1,2,3)$ du filtre dans les zones textes à prévoir sur le panneau de dialogue opérateur.

- Affichage des résultats d'acquisition de données et de filtrage dans un tableur.

- Représentation graphiquement des données.

- Sauvegarde des données acquises et traitées dans le disque au format Matlab.

- Initialisation du système instrumental.

- Fin de l'application logicielle.

\subsection{Panneau de dialogue opérateur et organigramme}

Après avoir esquissé manuellement un panneau de contrôle conforme aux spécifications définies dans la section 3.1, l'implémentation a été faite en Visual Basic en suivant les techniques de programmation système développées en détail dans [1] et [Int-3]. Puis, les procédures événementielles relatives aux objets visuels ont été implémentées. Étant donné que Visual basic ne dispose pas de fonction d'accès aux ports LPT d'un PC, il a été nécessaire de déclarer dans le programme source Visual Basic de la feuille de l'application, les lignes de code suivantes:

Private Declare Function Inp Lib "Inpout32.dll"

Alias "Inp32" (ByVal PortAddress As Integer) As Integer

\section{Private Declare Sub Out Lib "Inpout32.dll" Alias "Out32" (ByVal PortAddress As Integer, ByVal Value As Integer)}

Ces lignes de codes permettent ainsi d'exploiter les fonctions de lecture (Inp32) et d'écriture (Out32) au port $L P T$ d'un $P C$, contenues dans la bibliothèque de liens dynamiques INPOUT32.DLL disponible au site de téléchargement [Int-4]. L'organigramme de la figure 4 résume les spécifications fonctionnelles complètes du logiciel d'instrumentation virtuel proposé, conçu pour Windows 9.X/Me/2000/XP. L'aspect du panneau de dialogue opérateur à l'état initialisé correspond à la figure 5.

\section{Figure 4. Organigramme de fonctionnement du didacticiel}

\section{Figure 5. Panneau de dialogue opérateur à l'état initialisé}

\section{Travaux pratiques à l'aide du didacticiel}

Le mode opératoire d'une séance de travaux pratiques d'instrumentation par ordinateur basée sur le didacticiel proposé est le suivant :

\subsection{Préparation des travaux pratiques en deux parties}

- Étude de l'architecture et du fonctionnement de l'interface matérielle de génération et de conversion analogique-numérique de signaux générés. 
- Étude des principales procédures Visual Basic du logiciel d'instrumentation (conversion $\mathrm{A} / \mathrm{N}$, multiplexage, codes événementiels des composants du panneau de dialogue opérateur, etc.).

\subsection{Acquisition, traitement et sauvegarde des données}

- Connexion de la maquette (interface matérielle) à l'ordinateur et à la prise d'alimentation $220 \mathrm{~V} / 50 \mathrm{~Hz}$, puis mise sous tension.

- Lancement du logiciel d'instrumentation virtuel installé dans l'ordinateur.

- Fixation de la fréquence désirée à l'aide du potentiomètre gradué prévu sur la face avant de la maquette.

- Sélection de l'option signal triangulaire, puis clic sur Acquisition, et Visualisation.

- Sélection de l'option signal carré, puis clic sur Acquisition, et Visualisation.

- Sélection de l'option signal sinusoïdal, puis clic sur Acquisition, et Visualisation.

- Saisie des paramètres du filtre s'ils diffèrent des paramètres par défaut.

- Sélection du signal à filtrer, puis clic sur Filtre RII.

- Clic sur Sauvegarde pour sauvegarder dans le répertoire courant les données dans un fichier Matlab DAQLPT.M.

- Clic sur Quit si nécessaire pour sortir de l'application.

\subsection{Récupération des données avec Matlab}

Pour récupérer avec Matlab les données sauvegardées, il convient de procéder comme suit :

- Démarrage de Matlab.

- Définition comme répertoire courant celui dans lequel le fichier de données $D A Q L P T . M$ a été sauvegardé.

- Exécution du fichier DAQLPT, auquel cas le tableau de valeur sauvegardé est lu et devient disponible sous Matlab.

- Visualisation des données en tapant les lignes de code Matlab suivantes :

» $\operatorname{plot}(\operatorname{Tab}(:, 2), \operatorname{Tab}(:, 3), \operatorname{Tab}(:, 2), \operatorname{Tab}(:, 4), \operatorname{Tab}(:, 2), \operatorname{Tab}(:, 5))$, grid

» xlabel('(s)'), ylabel('V'), title('Matlab: Generation/Acquisition - ICL 8030')

»xlabel('(s)'), ylabel('Volts'), title('Matlab: Generation/Acquisition - ICL 8030')

- Traitement numérique avancé des données avec Matlab si nécessaire.

Les figures 6 à 9 présentent les copies d'écran des résultats attendus à divers stades du déroulement des travaux pratiques. La courbe ayant une allure exponentielle à la figure 6 , correspond au résultat de traitement du signal carré par un filtre RII (Réponse Impulsionnelle Infinie) dont les paramètres sont modifiables par l'opérateur.

Figure 6. Résultat d'acquisition des signaux : triangle, carré et sinus.

Figure 7. Sauvegarde des résultats obtenus.

Figure 8. Aspect du fichier de données DAQLPT.M sauvegardé.

Figure 9. Graphe des données récupérées et tracées avec Matlab. 


\section{Conclusion}

Le didacticiel proposé dans cet article est un produit original, initié et développé au sein du Groupe de Recherche en Informatique Industrielle et Automation de l'ENSET de Douala. Les premiers prototypes de ce produit d'appoint à la fois simple à mettre en œuvre et peu coûteux, nous ont permis de monter des postes de travaux pratiques d'instrumentation virtuelle et de traitement de signal en temps réel par ordinateur. Suite à la contribution didactique au niveau local de ce produit, nous nous intéressons actuellement à l'étude et à l'analyse d'un système de fabrication sérielle, qui pourra nous permettre d'étendre son utilisation à une plus large communauté technologique universitaire.

\section{Références bibliographiques}

[1] J. MBIHI, "Informatique et Automation: Automatismes Programmables Contrôlés par Ordinateur". Editions Publibook Universités, Paris, 2006.

[2] N. KELAKO KANEDJA, M. L. NGO NOUCK, "Conception et réalisation d'un système combiné GBF-Analyseur de signaux programmable par ordinateur". Mémoire de fin d'études en vue de l'obtention du DIPET-II, proposé et dirigé par J. MBIHI, GR2IA, ENSET de Douala, 2007.

\section{Sites Internet intéressants}

[Int-1] http://www.alldatasheet.com

[Int-2] http://crteknologies.free.fr/programmation/systeme/parallel.html

[Int-3] http://www.vbdeveloppez.com/

[Int-4] WWW.logic4u.cjb.net 
Figures citées dans le texte

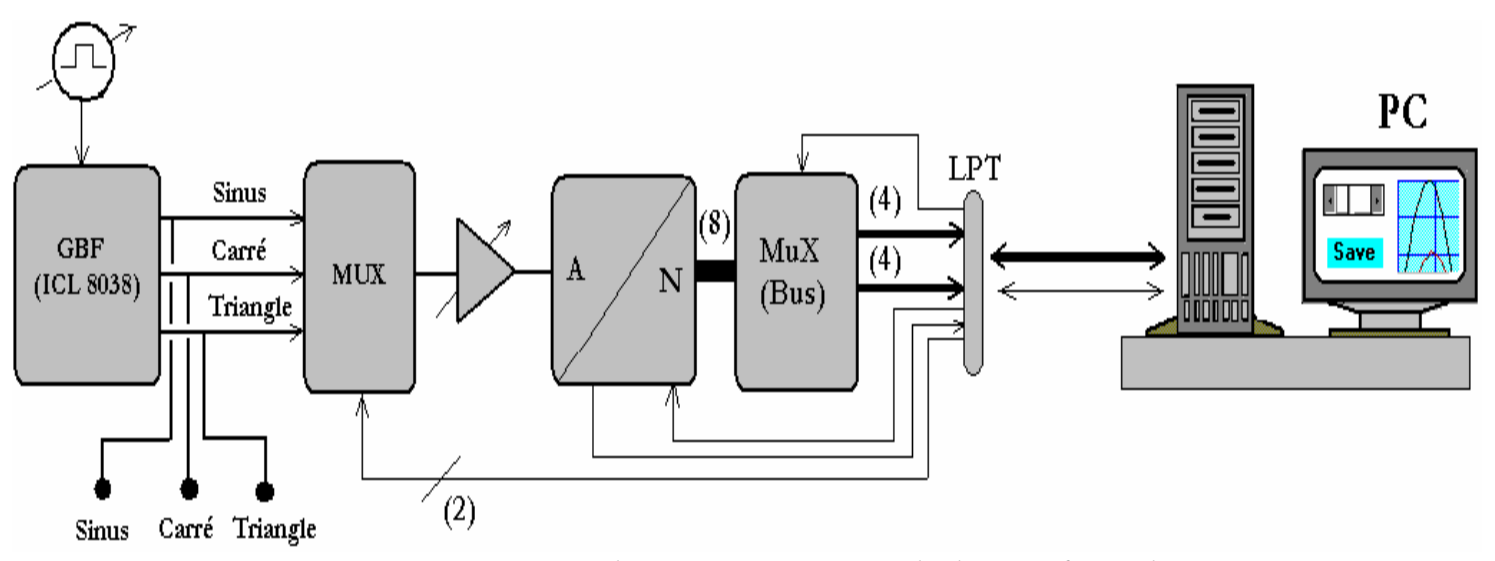

Figure 1 Schéma synoptique de l'interface d'entrée

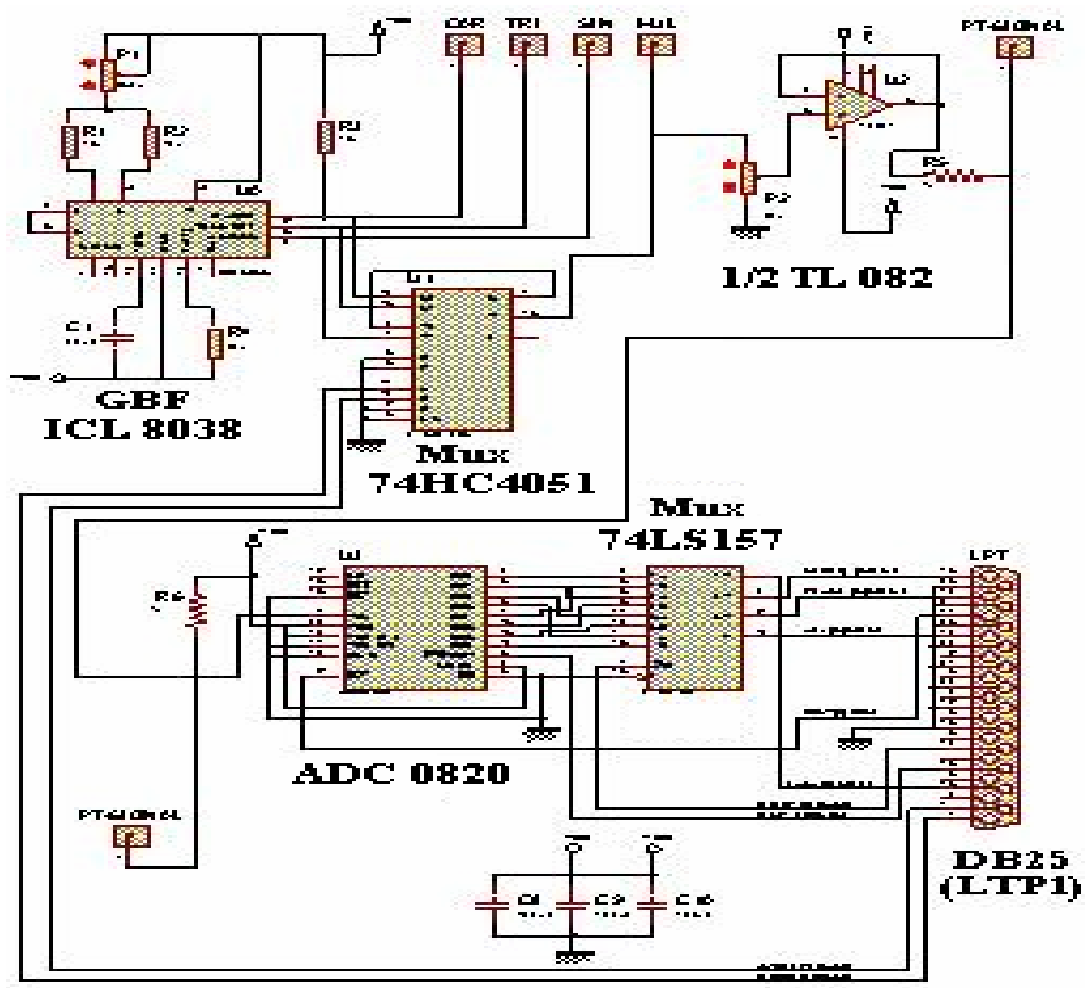

Figure 2 Schéma de principe de l'interface d'entrée 


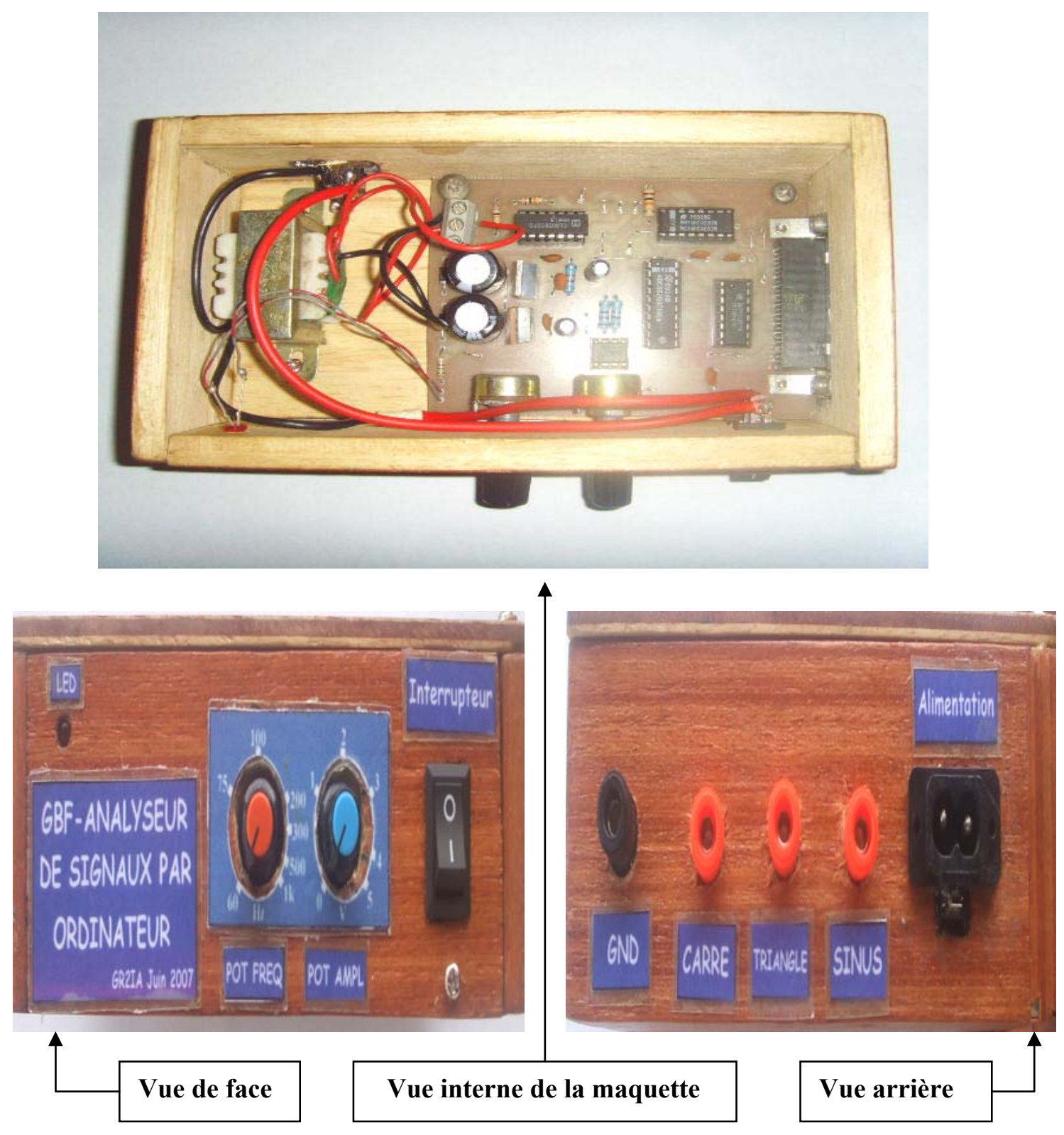

Figure 3 Prises de vue de la maquette didactique 


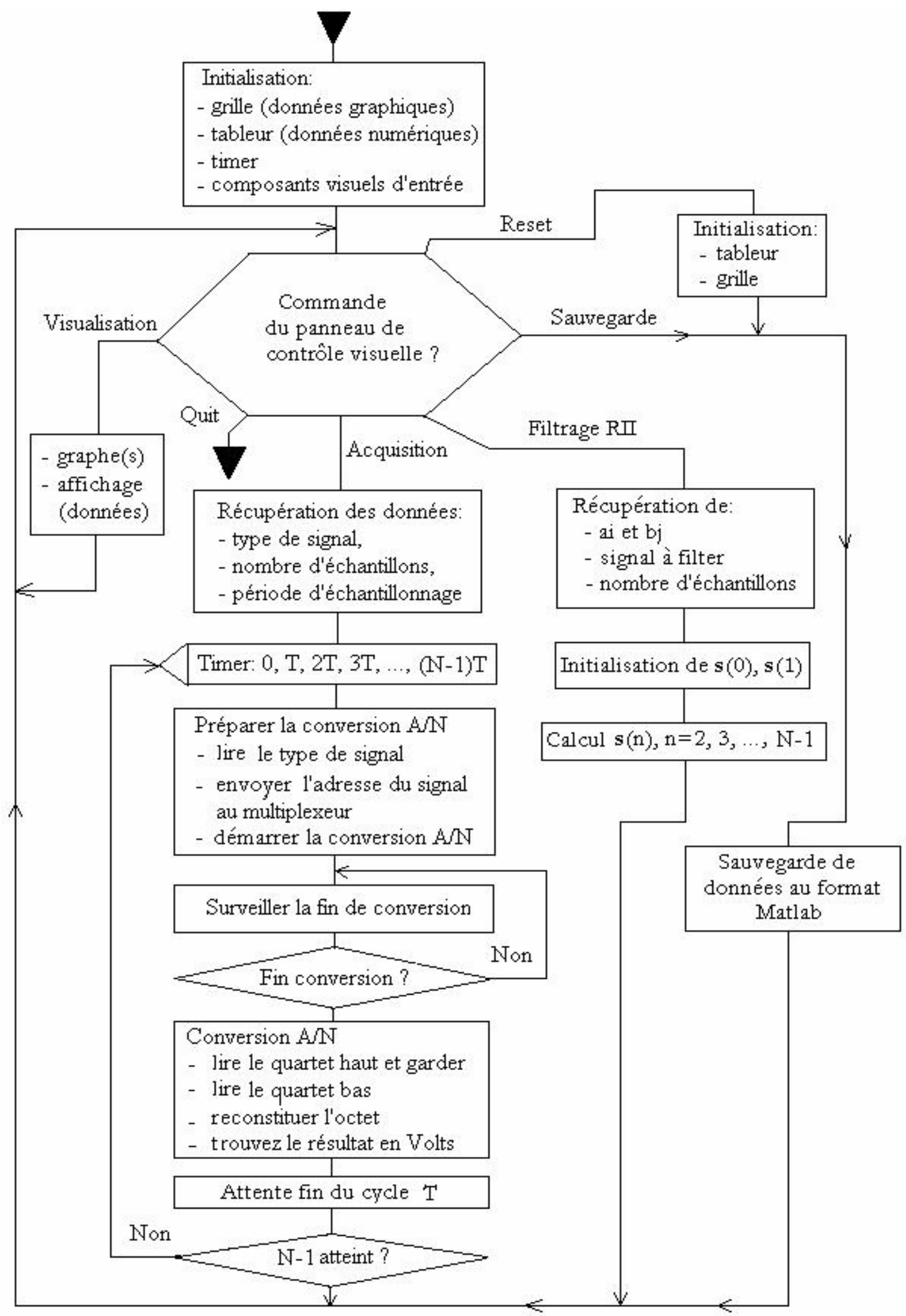

Figure 4 Organigramme de fonctionnement du didacticiel 


\begin{tabular}{|c|c|c|c|c|}
\hline $\mathrm{n}$ & $\mathrm{tn}=\mathrm{nT}[\mathrm{s}]$ & Triangle [V] & Carré(V] & $\Delta$ \\
\hline 0 & 0,0000 & 0,000 & 0 . & \\
\hline 1 & 0,0000 & 0,000 & 0 . & \\
\hline 2 & 0,0000 & 0,000 & 0 . & \\
\hline 3 & 0.0000 & 0,000 & 0 . & \\
\hline 4 & 0,0000 & 0,000 & 0 . & \\
\hline 5 & 0,0000 & 0,000 & 0 . & \\
\hline 6 & 0.0000 & 0,000 & 0 . & \\
\hline 7 & 0,0000 & 0,000 & 0 . & \\
\hline 8 & 0,0000 & 0,000 & 0 . & \\
\hline 9 & 0,0000 & 0,000 & 0 . & \\
\hline 10 & 0,0000 & 0.000 & 0 . & \\
\hline 11 & ก กกก & ก กกก & n & $\nabla$ \\
\hline $1 \square$ & & & 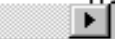 & \\
\hline
\end{tabular}
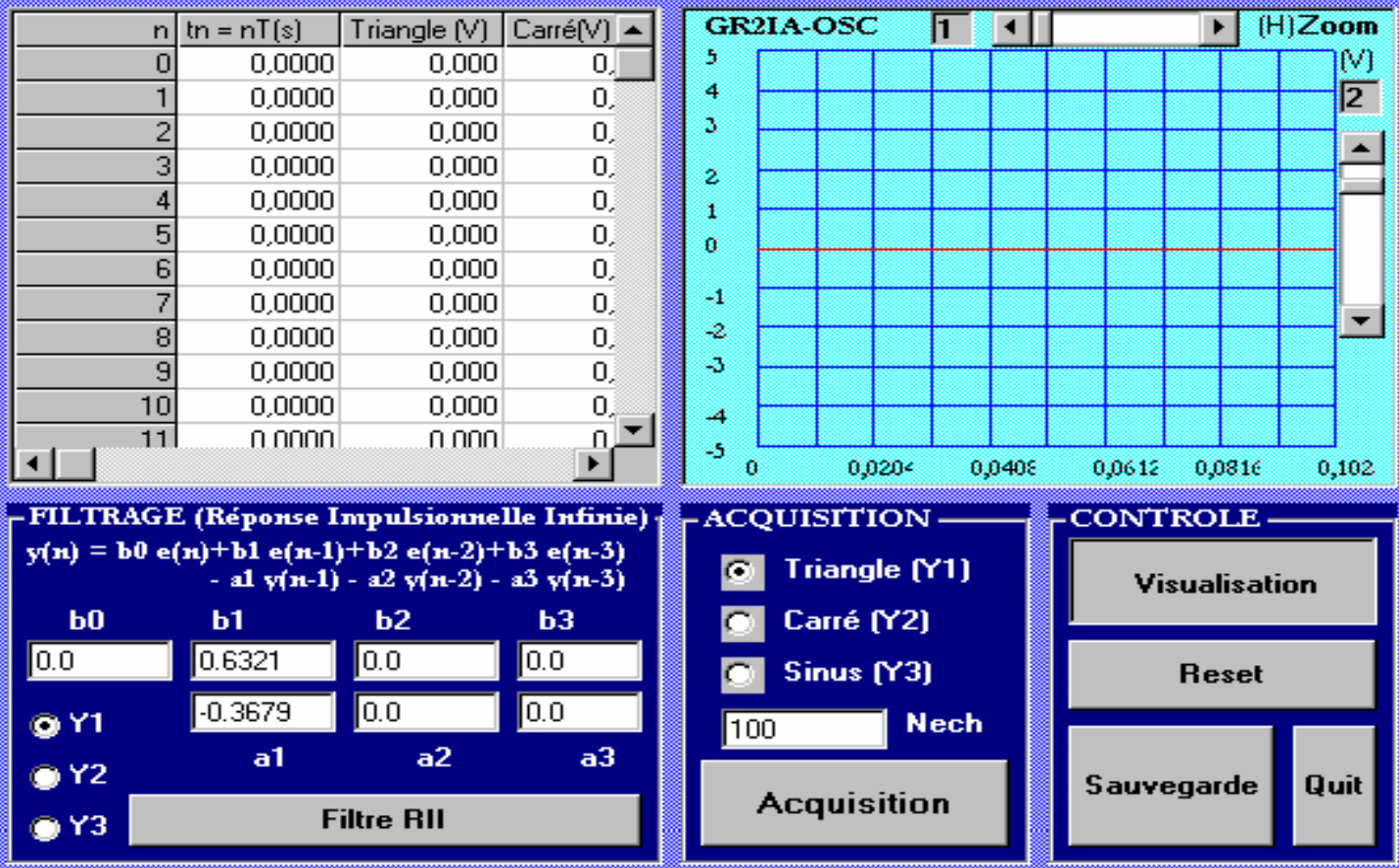

Figure 5 Panneau de dialogue opérateur à l'état initialisé

5. ADMON820

$-\square \times$

\begin{tabular}{|r|r|r|r|}
\hline $\mathrm{n}$ & $\mathrm{tn}=\mathrm{nT}[\mathrm{s}]$ & Triangle $\mathrm{V}$ ) & Carré(V) \\
\hline 0 & 0,0010 & 0,294 & 3, \\
\hline 1 & 0,0020 & 0,373 & 3, \\
\hline 2 & 0,0030 & 0,490 & 3 \\
\hline 3 & 0,0040 & 0,608 & 3 \\
\hline 4 & 0,0050 & 0,725 & 3 \\
\hline 5 & 0,0060 & 0,843 & 3 \\
\hline 6 & 0,0070 & 0,961 & 3 \\
\hline 7 & 0,0080 & 1,078 & 3 \\
\hline 8 & 0,0090 & 1,118 & 3 \\
\hline 9 & 0,0100 & 1,157 & 3 \\
\hline 10 & 0,0110 & 1,157 & 3 \\
\hline 11 & $n n 12 n$ & 1.118 & 3 \\
\hline
\end{tabular}

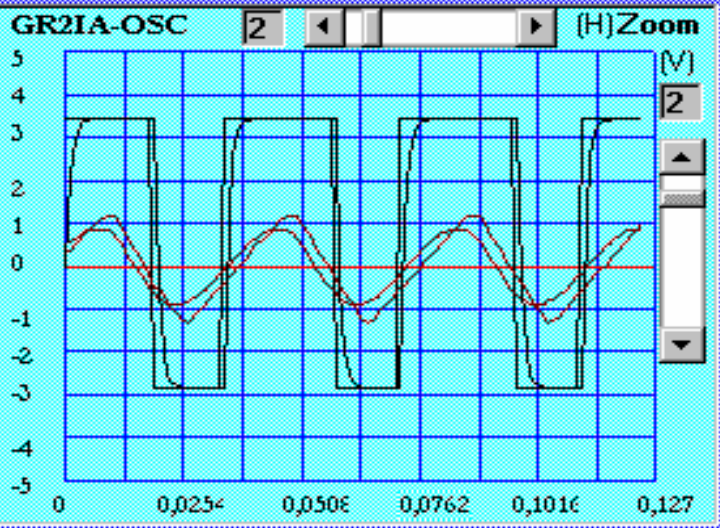

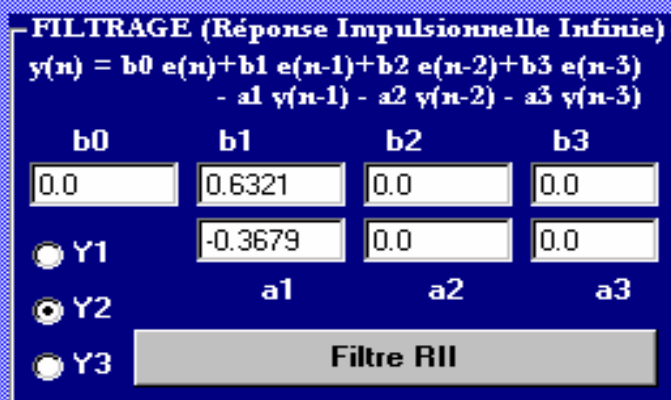
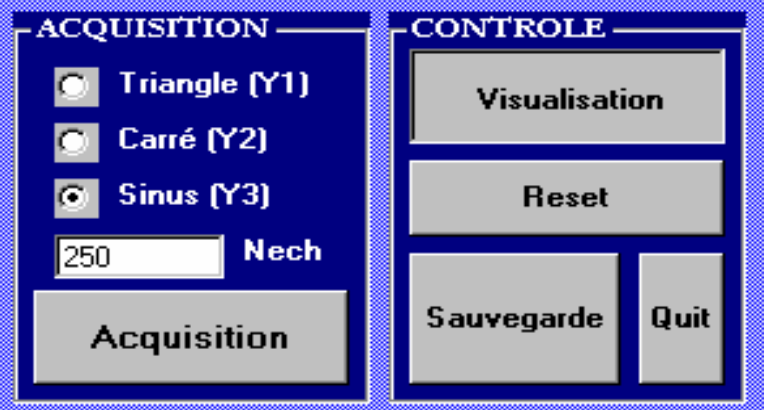

Figure 6 Résultats d'acquisition des signaux : triangle, carré et sinus. 


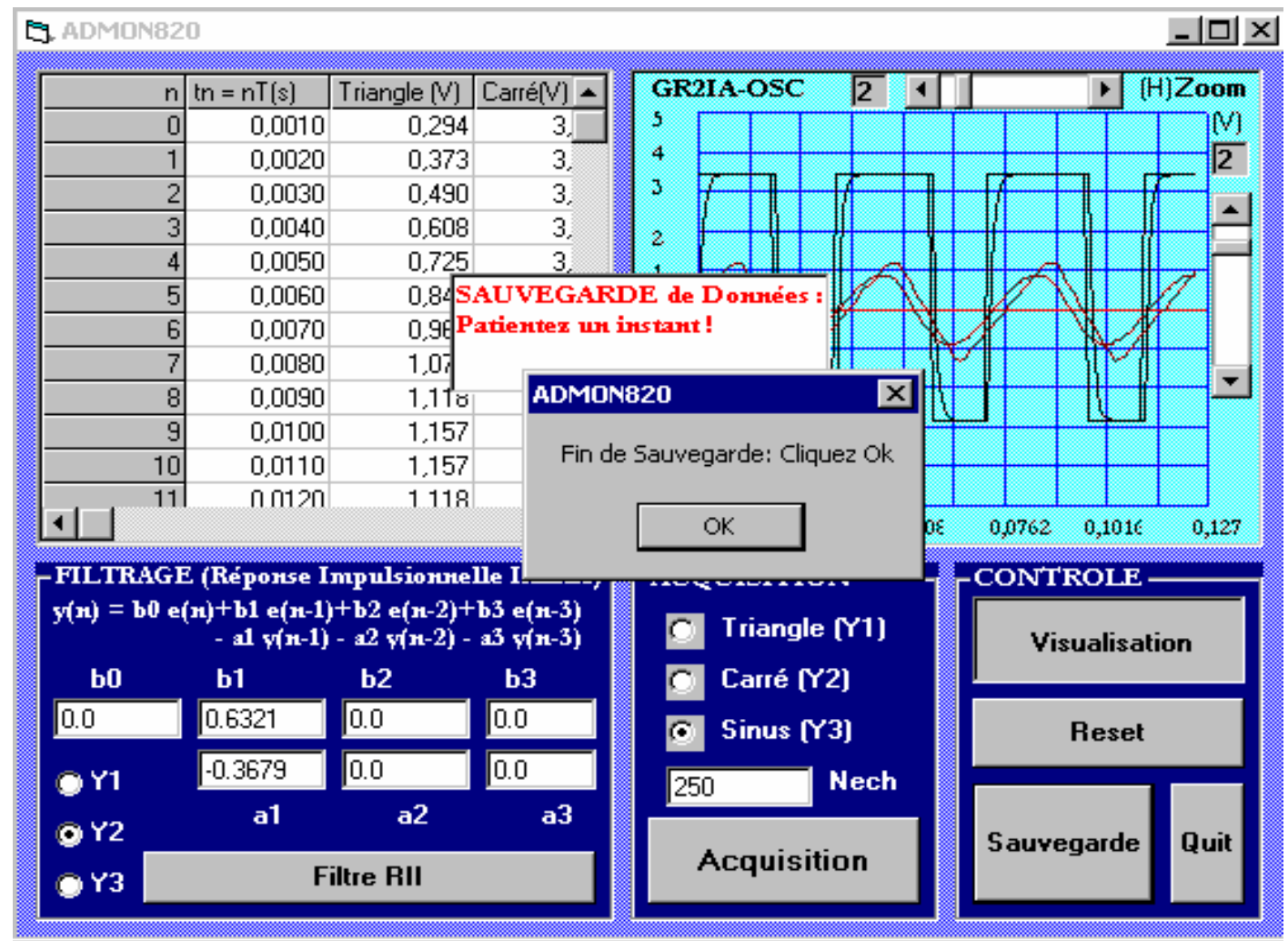

Figure 7 Sauvegarde des résultats

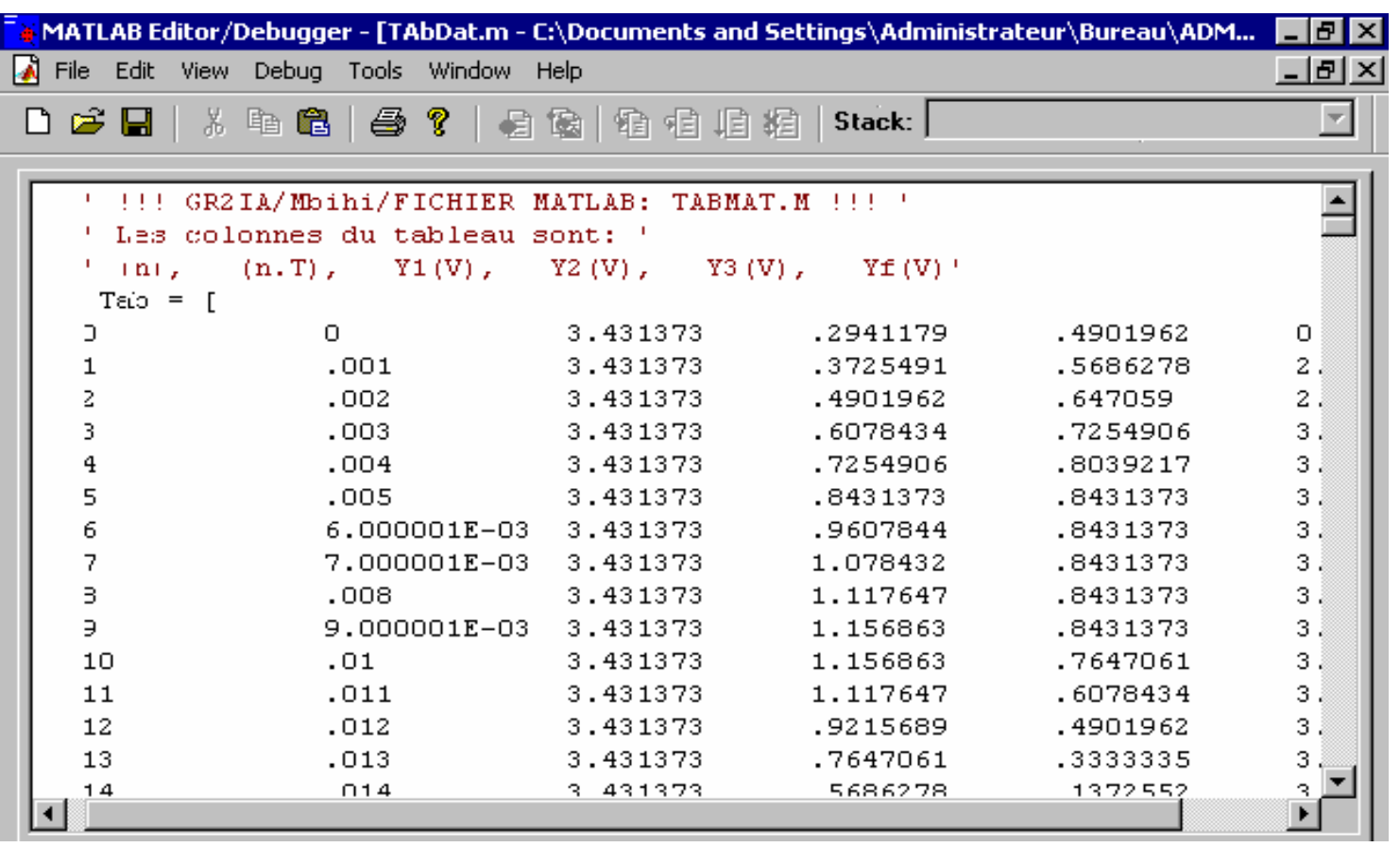

Figure 8 Aspect du fichier de données DAQLPT.M sauvegardé 


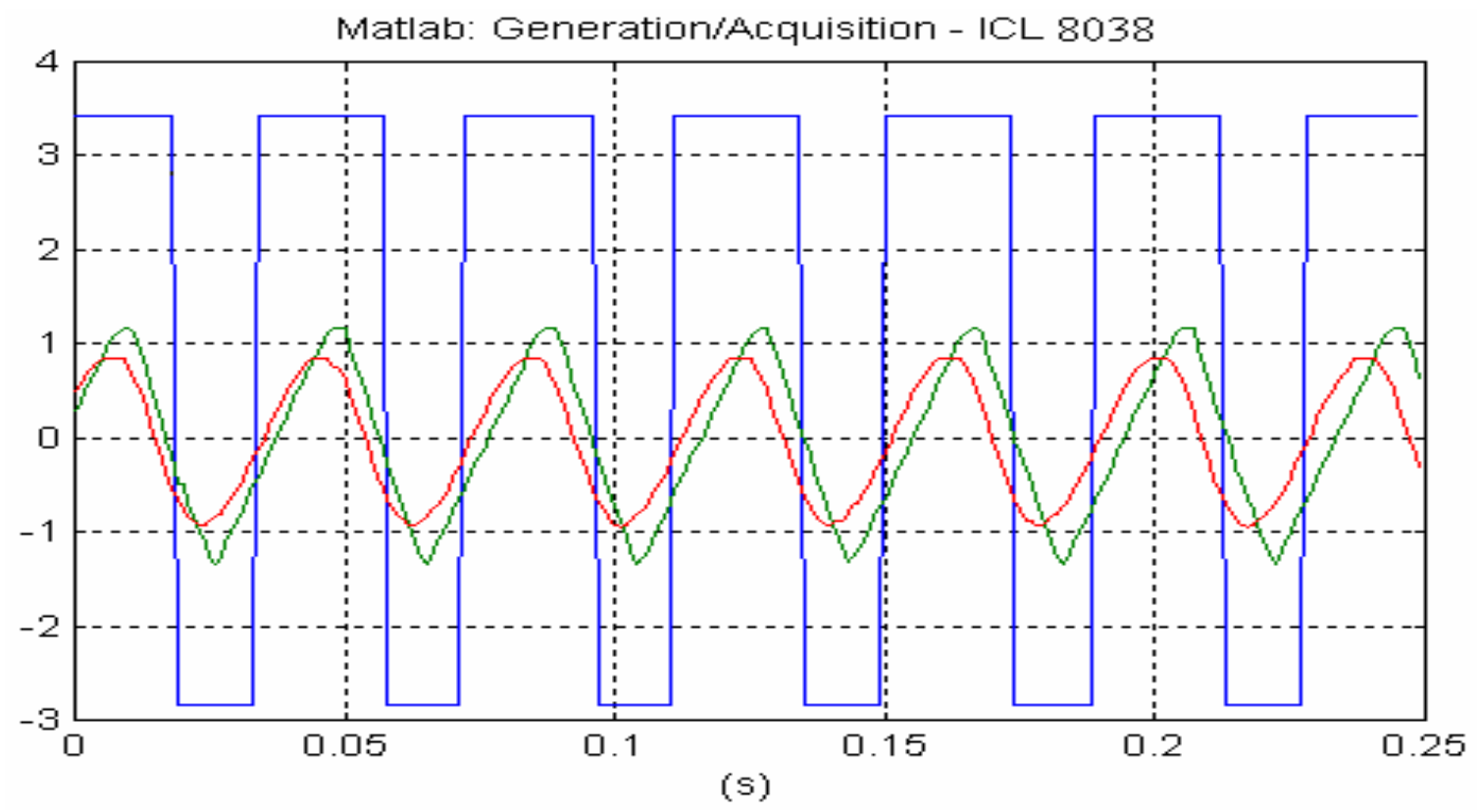

Figure 9 Données récupérées et tracées directement avec Matlab

\section{Biographie des auteurs :}

1) $\boldsymbol{J} . \boldsymbol{M B I H I}$ a obtenu le Ph.D. en génie électrique et informatique à l'École Polytechnique de Montréal (Québec, Canada) en 1999. Il est actuellement enseignant-chercheur, responsable du Groupe de Recherche en Informatique Industrielle et Automation (GR2IA), et chef du département d'Industries Textiles et de l'Habillement à l'ENSET de Douala. Il est membre du comité scientifique de l'unité de formation doctorale en Physique et Sciences de l'Ingénieur de l'Université de Douala, et membre du Comité Consultatif des Institutions Universitaires du Cameroun. Il est auteur de l'ouvrage Informatique et Automation, publié aux éditions Publibook de Paris (2006). Ses recherches actuelles portent sur les nouveaux systèmes d'instrumentation virtuelle et d'automation locale ou à distance.

2) N. KELAKO KENADJA est titulaire du Diplôme de Professeur d'Enseignement Technique (DIPET) de $2^{\mathrm{e}}$ grade en électrotechnique, obtenu à l'ENSET de Douala au Cameroun. Dans le cadre de la préparation de son mémoire de fin d'études pour l'obtention du DIPET 2, il a participé au sein du GR2IA aux travaux de conception, de montage et de mise en service de la partie matérielle du didacticiel présenté dans cet article.

3) M. L. NGO NOUCK est titulaire du Diplôme de Professeur d'Enseignement Technique (DIPET) de $2^{\mathrm{e}}$ grade en électrotechnique, obtenu à l'ENSET de Douala au Cameroun. Dans le cadre de la préparation de son mémoire de fin d'études pour l'obtention du DIPET 2, elle a participé au sein du GR2IA aux travaux de conception, de montage et de mise en service de la partie matérielle du didacticiel présenté dans cet article. 